\title{
NUTRIÇÃO FOSFATADA E RENDIMENTO DO FEIJOEIRO SOB INFLUÊNCIA DA CALAGEM E ADUBAÇÃO ORGÂNICA ${ }^{1}$
}

\author{
Phosphated nutrition and yield of the bean plant under the influence \\ of liming and organic fertilization
}

\author{
Renato Ferreira de Souza2 ${ }^{2}$,Valdemar Faquin ${ }^{3}$,Luis Arnaldo Fernandes ${ }^{4}$, \\ Fabricio Wiliam de Avila ${ }^{5}$
}

\begin{abstract}
RESUMO
Sendo um nutriente essencial em diversas funções celulares, o fósforo freqüentemente limita o desenvolvimento do feijoeiro devido sua alta capacidade de adsorção aos componentes mineralógicos do solo e sua baixa disponibilidade à absorção vegetal. Práticas de manejo do solo, como aumento do teor de matéria orgânica e a calagem, são bastante eficazes na redução do fenômeno de adsorção de P no solo e aumento de sua disponibilidade para a absorção vegetal. Objetivando avaliar o efeito de doses de esterco bovino e calcário sobre a nutrição fosfatada e produção do feijoeiro, foram conduzidos, em casa-de-vegetação do Departamento de Ciência do Solo da Universidade Federal de Lavras, quatro experimentos utilizando-se o delineamento inteiramente ao acaso, em esquema fatorial $5 \times 4$ e quatro repetições, em vasos contendo $3 \mathrm{dm}^{3}$ de amostras dos solos Neossolo Quartzarênico órtico, textura arenosa (RQo), Latossolo Vermelho-Amarelo distrófico, textura média (LVAd-1), Latossolo Vermelho-Amarelo distrófico, textura argilosa (LVAd-2) e Latossolo Vermelho distrófico, textura muito argilosa (LVd), onde foram cultivadas três plantas de feijoeiro. Os tratamentos foram constituídos pela aplicação de cinco doses de esterco bovino ( $0 ; 2,5 ; 5,0 ; 7,5$ e $10 \%$ do volume do solo) e quatro níveis de calagem ( 0,0 ; 0,$5 ; 1$ e 2 vezes a dose recomendada para atingir $\mathrm{V}=60 \%$ ). Foram avaliados a produção de matéria seca da parte aérea e grãos e o acúmulo de $\mathrm{P}$ na matéria seca da parte aérea total. A calagem e a adubação orgânica promoveram aumentos na absorção, acúmulo de $\mathrm{P}$ e produção de grãos pelo feijoeiro, o qual apresentou as melhores respostas com a dose $10 \%$ de esterco bovino combinada com o nível 1,0 de calagem.
\end{abstract}

Termos para indexação: Fósforo, calcário, adubação orgânica, feijão, Phaseolus vulgaris.

\begin{abstract}
Being an essential nutrient in several cellular functions, the phosphorus frequently limits the development of the bean plant due its high capacity of adsorption to mineralogical components of the soil and low availability for the plants absorption. Soil management practices, such as the increase in the level of organic matter and liming are quite effective in the reduction of the phenomenon of adsorption of $\mathrm{P}$ in the soil and increase of its availability for plant absorption. Aiming to evaluate the effect of doses of bovine manure and lime on the phosphate nutrition and production of the bean plant, four experiments were conducted in the greenhouse of the Soil Science Department of the Federal University of Lavras. The experimental design was completely randomized, in a $5 \times 4$ factorial scheme with four replications, in pots containing $3 \mathrm{dm}^{3}$ of soil samples from an Orthic Quartzarenic Neosol (RQo), a Dystrophic Red-Yellow Latosol, medium texture (LVAd-1), a Dystrophic Red-Yellow Latosol, clayey texture (LVAD-2), and a Dystrophic Red Latosol, very clayey texture $(\mathrm{LVd})$, in which three bean plants were cultivated. The treatments were constituted by the application of five doses of bovine manure $(0 ; 2.5 ; 5.0 ; 7.5$, and $10 \%$ of the volume of the soil) and four liming levels $(0 ; 0.5 ; 1$, and 2 times the recommended dose to reach $\mathrm{V}=60 \%$ ). It were evaluated the production of dry matter of aerial part and grains and the $\mathrm{P}$ accumulation in dry matter of total aerial parts of beans plants. The liming and organic fertilization promoted increases in the absorption and accumulation of $\mathrm{P}$, and production of grains of the bean plant, which presented the best responses with the dose of $10 \%$ of bovine manure combined with the level 1 of liming.
\end{abstract}

Index terms: Phosphorus, limestone, manuring, bean plant, Phaseolus vulgaris.

(Recebido para publicação em 22 de março de 2005 e aprovado em 7 de dezembro de 2005)

\section{INTRODUÇÃO}

O feijoeiro (Phaseolus vulgaris L.) é uma das culturas mais difundidas no Brasil, tendo, além do caráter econômico, um alto significado social, por ser um alimento básico para a população. Entretanto, apesar de sua importância, ainda apresenta uma produtividade relativamente baixa. Os aspectos relacionados à baixa fertilidade e presença de elementos tóxicos no solo despontam como dos mais limitantes fatores responsáveis pela baixa produtividade da cultura no Brasil.

${ }^{1}$ Parte da Tese de autoria do primeiro autor para a obtenção do Título de Doutor em Agronomia/Solos e Nutrição de Plantas.

${ }^{2}$ Doutor em Solos e Nutrição de Plantas - Universidade Federal de Lavras/UFLA - Departamento de Ciência do Solo/DCS - Cx. P. 3037 - $37200-000$ Lavras, MG - souzarf@ufla.br

${ }^{3}$ Professor Titular - Universidade Federal de Lavras/UFLA - Departamento de Ciência do Solo/DCS - Cx. P. 3037 - $37200-000$ - Lavras, MG

${ }^{4}$ Professor do NCA - Universidade Federal de Minas Gerais/UFMG.

${ }^{5}$ Acadêmico de Agronomia - Universidade Federal de Lavras/UFLA - Bolsista de I.C./CNPq. Projeto financiado pela FAPEMIG e CNPq. 
O fósforo é um elemento essencial ao crescimento e reprodução das plantas, as quais não alcançam seu máximo potencial produtivo sem um adequado suprimento nutricional (MARSCHNER, 1995). O fósforo é componente de compostos importantes das células vegetais, incluindo fosfato presente nas moléculas de açúcares, intermediários da respiração e fotossíntese, bem como dos fosfolipídeos que compõem as membranas vegetais. É, também, componente de nucleotídeos utilizados no metabolismo energético das plantas (como ATP) e dos ácidos nucléicos (TAIZ \& ZEIGER, 2004).

A influência do suprimento de fósforo sobre a produção do feijoeiro tem sido estudada em diversos trabalhos, citando-se Fageria \& Santos (1998), Oliveira et al. (1987) e Parra \& Miranda (1980) confirmando a grande importância desse nutriente para a cultura, embora seja, entre os macronutrientes, o menos exigido. Entretanto, esse nutriente é considerado de maior limitação nutricional ao desenvolvimento das culturas nos solos das regiões tropicais. Isso é devido à alta fixação do $\mathrm{P}$ aos componentes mineralógicos do solo, concorrendo para os valores extremamente baixos de fósforo em solução (P-disponível). Embora o teor total de P no solo se situe entre 200 e 3000 $\mathrm{mg} \mathrm{kg}^{-1}$, na maioria das vezes, menos de $0,1 \%(0,2 \mathrm{a} 3 \mathrm{mg}$ $\mathrm{kg}^{-1}$ ) desse fósforo se encontra em solução, ou seja, prontamente disponível à absorção vegetal (NOVAIS \& SMYTH, 1999). Isso ocorre, pois, os solos das regiões tropicais são geralmente ácidos e apresentam quantidades significativas de oxidróxidos de $\mathrm{Fe}$ e $\mathrm{Al}$ e caulinita, carregados positivamente, apresentando alta capacidade de fixação de fosfato, que é adsorvido às superfícies dos colóides por um complexo de esfera interna (MEURER et al., 2004).

A calagem e a interação da matéria orgânica (MO) com os argilominerais e óxidos do solo alteram o balanço de cargas elétricas superficiais. A MO é adsorvida à caulinita e aos oxidróxidos de $\mathrm{Fe}$ e $\mathrm{Al}$, resultando na redução das cargas positivas. A quantidade e a proporção de cargas elétricas no solo está condicionada ao $\mathrm{pH}$ da solução, havendo elevação das cargas negativas e, conseqüentemente, redução do fenômeno de adsorção de $\mathrm{P}$, com o aumento do $\mathrm{pH}$ por meio da calagem (MEURER et al., 2004). Portanto, as práticas de manejo do solo, tais como aumento do teor de matéria orgânica e calagem são bastante eficazes na redução do fenômeno de fixação de P. A menor adsorção e, conseqüentemente, maior disponibilidade de fósforo no solo resulta em uma melhor nutrição do feijoeiro e, conseqüente, redução no uso de fertilizantes fosfatados.
Objetivou-se com este estudo avaliar o efeito da calagem e aplicação de esterco bovino sobre a nutrição fosfatada e produção do feijoeiro (Phaseolus vulgaris $\mathrm{L}$.) cultivado em quatro solos com texturas, mineralogia e teores de matéria orgânica bastante distintos.

\section{MATERIAL E MÉTODOS}

Para abranger uma ampla variação nas características físicas, químicas e mineralógicas, foram utilizados os solos classificados no Sistema Brasileiro de Classificação de Solos (EMBRAPA, 1999) como: Neossolo Quartzarênico órtico, textura arenosa (RQo, coordenadas UTM: x: 0540201 e y: 7644073 - 23K; Itutinga - MG), Latossolo Vermelho-Amarelo distrófico, textura média (LVAd-1, coordenadas UTM: x: $0521106 \mathrm{e}$ y: 7646097 - 23K; Itumirim - MG), Latossolo VermelhoAmarelo distrófico, textura argilosa (LVAd-2, coordenadas UTM: x: 0504202 e y: 7652821 - 23K; Lavras - MG) e Latossolo Vermelho distrófico, textura muito argilosa (LVd, coordenadas UTM: x: 0503646 e y: 7652507 - 23K; Lavras - MG).

Os solos foram coletados na camada de $0-20 \mathrm{~cm}$ de profundidade, sob vegetação natural, tomando-se o cuidado de retirar a vegetação e restos orgânicos presentes na superfície. Após tamisagem em malha de $4 \mathrm{~mm}$, subamostras dos solos foram tomadas para caracterizações químicas, físicas e mineralógicas (Tabela 1).

Quatro experimentos foram conduzidos, em casade-vegetação do Departamento de Ciências do Solo da Universidade Federal de Lavras (UFLA), utilizando-se o delineamento experimental inteiramente ao acaso (DIC), com os tratamentos em esquema fatorial $4 \times 5 \mathrm{com}$ quatro repetições, em vasos com capacidade de três $\mathrm{dm}^{3}$ de solo. Os tratamentos foram constituídos de cinco doses de esterco bovino curtido, correspondentes a 0,0 ; 2,$5 ; 5,0 ; 7,5$ e $10 \%$ do volume de solo de cada parcela experimental e quatro níveis de calagem, equivalentes a 0,$0 ; 0,5 ; 1,0$ e 2,0 vezes as doses recomendadas para elevar a saturação por bases em cada solo a $60 \%$, segundo CFSEMG (1999). Utilizou-se um calcário dolomítico calcinado e micropulverizado, com $35 \%$ de $\mathrm{CaO}, 14 \%$ de $\mathrm{MgO}$ e PRNT de $100 \%$. O esterco bovino foi moído ( $1 \mathrm{~mm}^{2}$ de dimensão) e seco a $60{ }^{\circ} \mathrm{C}$ e após sua incorporação, juntamente com as doses de calcário, o solo de cada unidade experimental, foi incubado por um período de 30 dias, mantendo-se a umidade próxima à capacidade de campo. 
TABELA 1 - Principais atributos químicos, físicos e mineralógicos de amostras dos solos, sob condições naturais, coletadas na camada de 0 a $20 \mathrm{~cm}$.

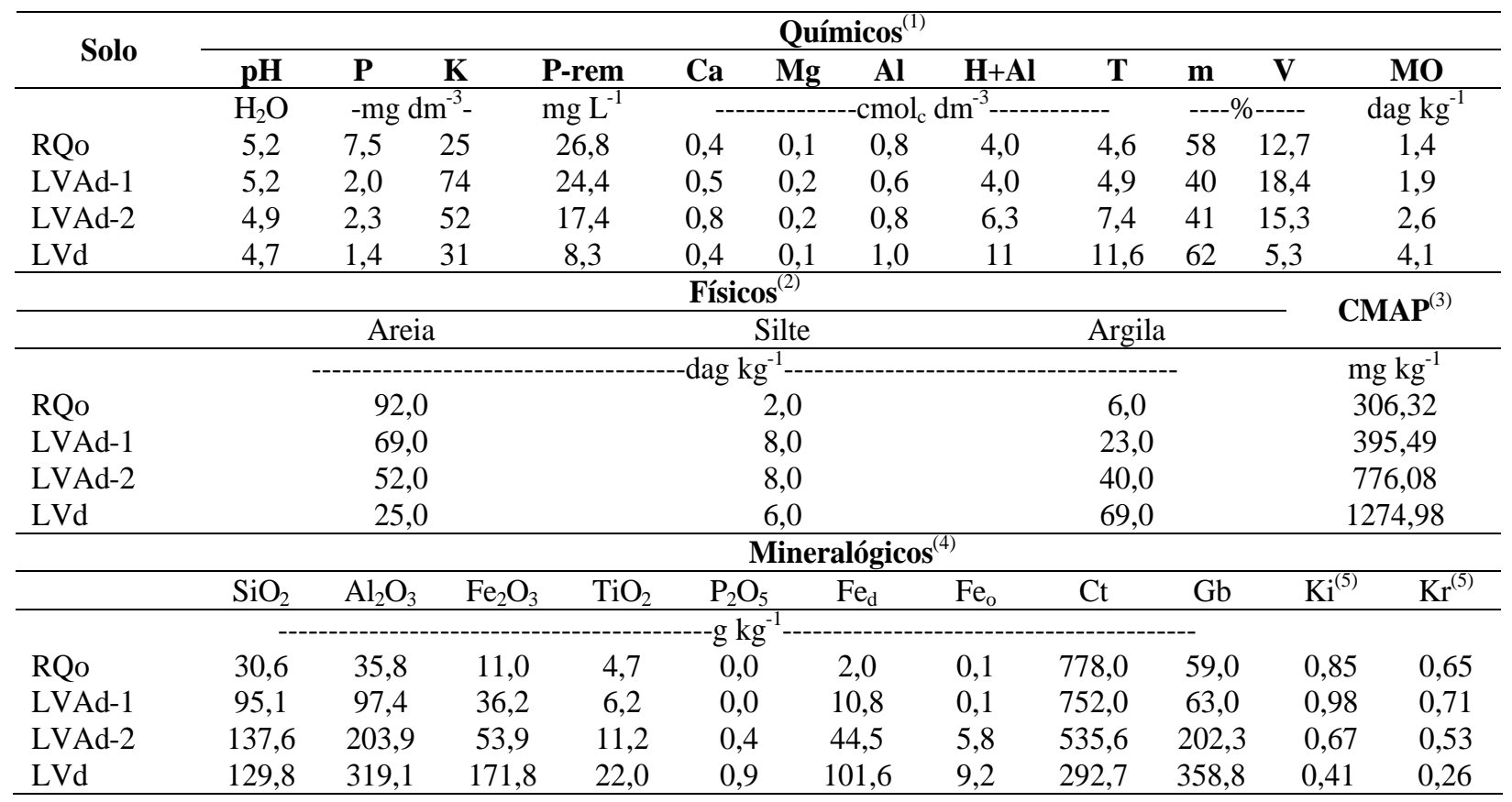

${ }^{(1)} \mathrm{pH}$ em água, $\mathrm{Ca}, \mathrm{Mg}, \mathrm{Al}, \mathrm{P}$ (Mehlich-1), K foram determinados conforme Embrapa (1997); Fósforo remanescente foi determinado conforme Alvarez et al. (2000); H+Al e teor de carbono orgânico, conforme Raij et al. (1987); T = Capacidade de troca catiônica a pH 7,0; $\mathrm{m}$ = Índice de saturação de alumínio e V = Índice de saturação de bases;

(2) A granulometria dos solos foi determinada pelo método da pipeta (DAY, 1965).

${ }^{(3)}$ CMAP: capacidade máxima de adsorção de $\mathrm{P}$.

(4) $\mathrm{SiO}_{2}, \mathrm{Al}_{2} \mathrm{O}_{3}, \mathrm{Fe}_{2} \mathrm{O}_{3}, \mathrm{TiO}_{2}$ e $\mathrm{P}_{2} \mathrm{O}_{5}$ foram determinados conforme Embrapa (1997); $\mathrm{Fe}_{\mathrm{d}}$, segundo Mehra \& Jackson (1960) e $\mathrm{Fe}_{\mathrm{o}}$, segundo Schwertmann (1964); Ct (caulinita) e Gb (gibbsita) conforme Klug \& Alexander (1974).

${ }^{(5)}$ Relações moleculares: $\mathrm{Ki}=\mathrm{SiO}_{2} / \mathrm{Al}_{2} \mathrm{O}_{3}$ e $\mathrm{Kr}=\mathrm{SiO}_{2} / \mathrm{Al}_{2} \mathrm{O}_{3}+\mathrm{Fe}_{2} \mathrm{O}_{3}$.

Ao final desse período de incubação, foram coletadas subamostras de solo de cada parcela experimental, nas quais foram determinados: o P Mehlich1, o $\mathrm{P}$ remanescente (P-rem), a capacidade máxima de adsorção de $\mathrm{P}$ (CMAP). As metodologias, bem como os resultados dessas determinações estão em Souza (2005), os quais foram utilizados para estudos de correlações com as variáveis avaliadas no presente trabalho.

Após a incubação com os tratamentos, os solos LVd, LVAd-1, LVAd-2 e RQo, receberam, respectivamente, as doses de 400, 320, 250 e $200 \mathrm{mg} \mathrm{dm}^{-3}$ de $\mathrm{P}$ na forma de $\mathrm{Ca}\left(\mathrm{H}_{2} \mathrm{PO}_{4}\right)_{2} \cdot \mathrm{H}_{2} \mathrm{O}$, seguindo a sugestão de Alvarez \& Fonseca (1990). Os solos receberam, ainda, a aplicação de uma adubação básica com macro e micronutrientes, que forneceu: $70 \mathrm{mg}$ de $\mathrm{N} ; 100 \mathrm{mg}$ de $\mathrm{K} ; 40 \mathrm{mg}$ de $\mathrm{S} ; 0,5$ $\mathrm{mg}$ de $\mathrm{B} ; 1,5 \mathrm{mg}$ de $\mathrm{Cu}$ e 5,0 $\mathrm{mg}$ de $\mathrm{Zn} \mathrm{dm}^{-3}$ de solo, na forma de reagentes p.a.. Os nutrientes foram aplicados em forma de solução e misturadas ao solo para melhor uniformização. As fontes utilizadas foram: $\mathrm{KH}_{2} \mathrm{PO}_{4}$; $\mathrm{MgSO}_{4} \cdot 7 \mathrm{H}_{2} \mathrm{O} ; \mathrm{Mg}\left(\mathrm{NO}_{3}\right)_{2} \cdot 6 \mathrm{H}_{2} \mathrm{O} ; \mathrm{Ca}\left(\mathrm{NO}_{3}\right)_{2} \cdot 4 \mathrm{H}_{2} \mathrm{O}$; $\mathrm{CuCl}_{2} \cdot 2 \mathrm{H}_{2} \mathrm{O} ; \mathrm{ZnSO}_{4} \cdot 7 \mathrm{H}_{2} \mathrm{O} ;$ e $\mathrm{H}_{3} \mathrm{BO}_{3}$. As amostras dos solos dos vasos foram novamente incubadas, com umidade próxima à capacidade de campo, por mais 30 dias. Foram conduzidas, em cada vaso, três plantas de feijoeiro (Phaseolus vulgaris L. cv. Carioca MG). Durante o ciclo da cultura foram aplicadas adubações em cobertura nas doses de $300 \mathrm{mg} \mathrm{dm}^{-3} \mathrm{de} \mathrm{N}, 250 \mathrm{mg} \mathrm{dm}^{-}$ ${ }^{3}$ de $\mathrm{K} \mathrm{e} 90 \mathrm{mg} \mathrm{dm}^{-3}$ de $\mathrm{S}$, parceladas em cinco vezes até a fase de florescimento. Durante o período experimental, a umidade dos solos foi mantida em $60 \%$ do volume total de poros (FREIRE et al., 1980) utilizando água deionizada. 
Na colheita, a parte aérea do feijoeiro foi separada em caules + folhas e grãos, seco em estufa de circulação forçada de ar, à temperatura entre 65 e $70^{\circ} \mathrm{C}$ e pesada para a avaliação da produção da matéria seca da parte aérea (MSPA) e de grãos (MSGR). Na MSPA e MSGR, foi determinada a concentração de $\mathrm{P}$ de acordo com Malavolta et al. (1997) e a leitura nos extratos foi feita por colorimetria, segundo Braga \& Defelipo (1974). Relacionando-se as concentrações de $\mathrm{P}$ com a produção de matéria seca, determinou-se o P-acumulado na parte aérea total do feijoeiro.

Para as variáveis estudadas foram ajustados modelos de regressão múltipla (superfícies de resposta) em função das doses de calcário e de esterco bovino (ALVAREZ, 1994; KHURI \& CORNELL, 1996). Foi utilizado o programa de análises estatísticas R (R DEVELOPMENT CORE TEAM, 2004).

\section{RESULTADOS E DISCUSSÃO}

A adição de matéria orgânica e calagem afetaram significativamente o desenvolvimento do feijoeiro, promovendo uma maior produção de matéria seca da parte aérea e produção de grãos. Em geral, em todos os solos, ocorreram as maiores produções de grãos com as doses altas de matéria orgânica. Na Figura 1 e Tabelas 2 e 3 verificam-se que as maiores produções de grãos ocorreram nas seguintes doses de matéria orgânica (MO) e níveis de calagem (CAL): 5,28 (ou 9,76) \% de MO e 2,0 (ou 1,0) vez CAL para o solo RQo; $10 \%$ MO e 0,24 vez CAL para o solo LVAd-1; $10 \%$ e 2,0 vezes CAL para o solo LVAd-2 e 7,8 \% e 2,0 vezes CAL para o solo LVd.

$\mathrm{O}$ aumento da produtividade do feijoeiro, com a aplicação de esterco bovino e calcário, se deve, provavelmente, à maior disponibilidade de $\mathrm{P}$ via redução de sua fixação através do bloqueio dos sítios de adsorção do nutriente no solo. Andrade et al. (2002), Faquin et al. (1998) e Fernandes et al. (2002) em solos de várzea verificaram que o feijoeiro é responsivo à aplicação de calcário, sendo esse corretivo essencial para fornecer $\mathrm{Ca}$, $\mathrm{Mg}$, elevar o $\mathrm{pH}$ e neutralizar a toxidez por $\mathrm{Al}$ e $\mathrm{Mn}$.

$\mathrm{O}$ acúmulo total de $\mathrm{P}$ na parte aérea do feijoeiro (MSPA + MSGR) também foi influenciado pela aplicação de calcário e esterco bovino nos quatro solos. Os maiores acúmulos foram observados nas seguintes doses de matéria orgânica (MO) e níveis de calagem (CAL): $10 \%$ MO e 0,48 vez CAL para o solo RQo; $10 \%$ MO e 0,99 vez CAL, para o solo LVAd- $1 ; 10 \%$ MO e 0,50 vez CAL para o solo LVAd-2 e $10 \%$ MO e 2 vezes CAL para o solo LVd (Figura 1 e Tabelas 2 e 3).
A adição de esterco bovino e a calagem, alterando os atributos ligados ao fator capacidade de $\mathrm{P}$ dos solos (CMAP e P-rem), aumentou a disponibilidade do nutriente (SOUZA, 2005), favorecendo maior absorção de fósforo pelo feijoeiro e, conseqüentemente, maior acúmulo do nutriente e incremento na produção de grãos.

Verificou-se que a produção de grãos pelo feijoeiro foi diretamente proporcional ao P-rem e inversamente proporcional à CMAP (Tabela 4) (SOUZA, 2005), que representam a capacidade do solo em repor o P em solução. Considerando que o grão é a parte comercial do feijoeiro, a aplicação de resíduos orgânicos e a calagem podem maximizar a produção pela redução da adsorção de $\mathrm{P}$ no solo e sua maior disponibilidade para a planta.

$\mathrm{O}$ maior acúmulo de $\mathrm{P}$ foi observado nas plantas cultivadas nos solos mais argilosos, com menores valores de P-rem e maiores CMAP (SOUZA, 2005). Isso ocorreu devido à maior capacidade de armazenamento de $\mathrm{P}$ dos solos argilosos (NOVAIS \& SMYTH, 1999). Segundo esse mesmo autor, a maior ou menor competição entre plantas e solo pelo $\mathrm{P}$ aplicado faz com que a planta se ajuste para utilizar o $\mathrm{P}$ que lhe é colocado à disposição. A capacidade máxima de adsorção (CMAP) e o P-rem são atributos que refletem o poder tampão ou fator capacidade de $\mathrm{P}$ no solo (MUNIZ et al., 1985). E a variação na adsorção de P entre os solos normalmente está associada à mineralogia, textura e matéria orgânica do solo (BAHIA FILHO et al., 1983; MUNIZ et al., 1985). Vários autores observaram relação inversa entre o $\mathrm{P}$ absorvido pela planta e o fator capacidade de P do solo (BAHIA FILHO et al., 1983; FABRES et al., 1987; FERNANDES et al., 1998; HOLFORD \& MATTINGLY, 1976; MUNIZ et al., 1983). Porém, o maior conteúdo da fração de $\mathrm{P}$ pouco lábil apresentado pelos solos mais argilosos (SOUZA, 2005) supriu o P em solução, concorrendo para maior absorção do nutriente e maior desenvolvimento vegetal e, conseqüentemente, maior acúmulo do nutriente e produção pelas plantas.

Para o mesmo solo, o acúmulo de $\mathrm{P}$ na parte aérea total foi inversamente proporcional à CMAP e diretamente proporcional aos atributos P-rem e P Melhich-1, conforme pode ser observado na Tabela 4. Dessa forma, quando o $P$ é o único fator limitante à produção, além das variações decorrentes entre espécies ou cultivares, idade do tecido vegetal, forma do nutriente, interação entre nutrientes e condições ambientais, a CMAP deve ser considerada na interpretação dos conteúdos de P nas plantas (MUNIZ et al., 1985). Andrade et al. (2002) verificaram correlação positiva entre teor foliar de $\mathrm{P}$ e P-rem e negativa entre $\mathrm{o}$ teor foliar e CMAP, para solos de várzea. 

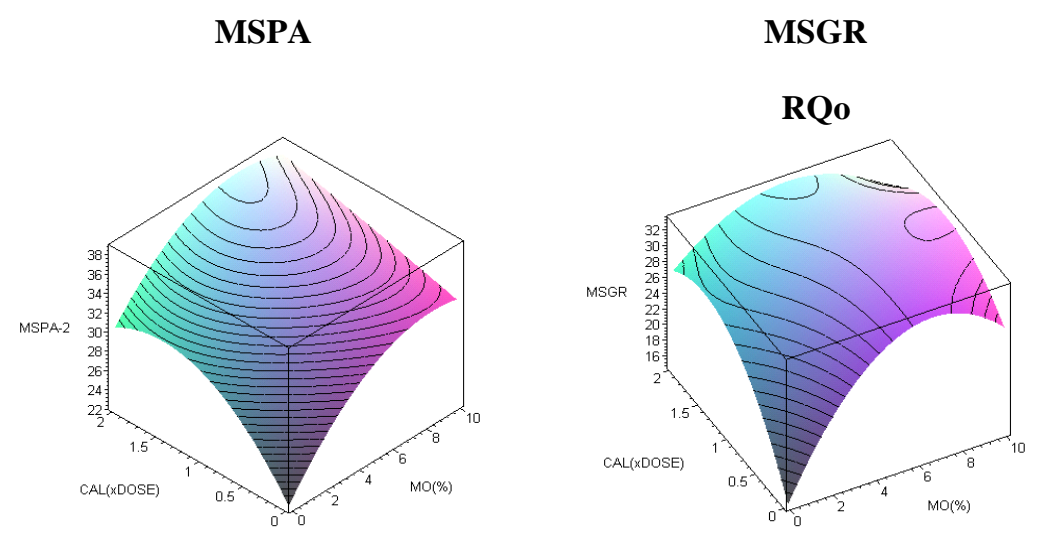

\section{Acúmulo de $\mathbf{P}$}

\section{LVAd-1}
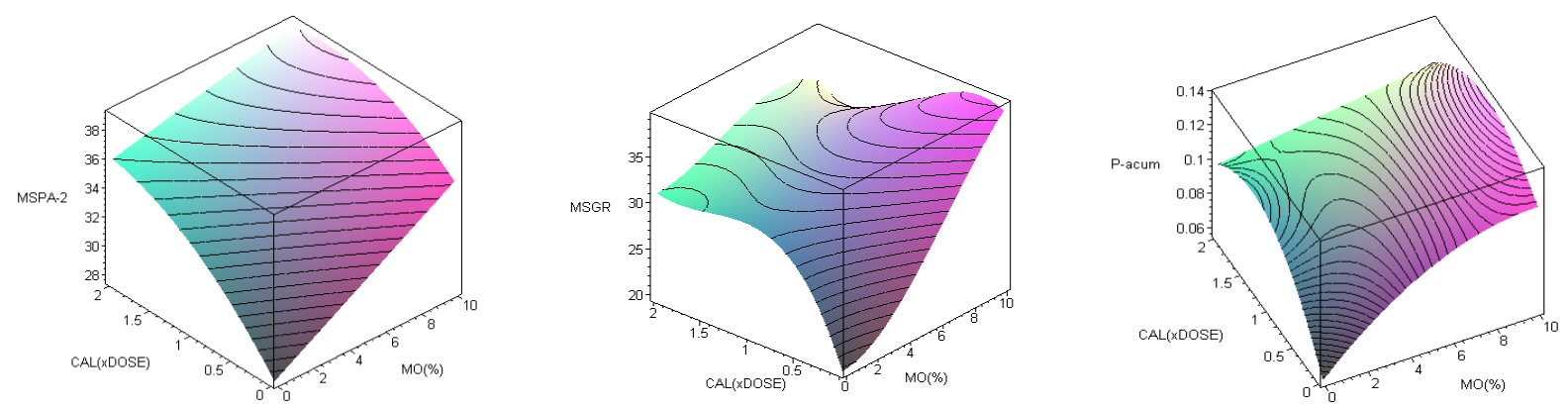

\section{LVAd-2}
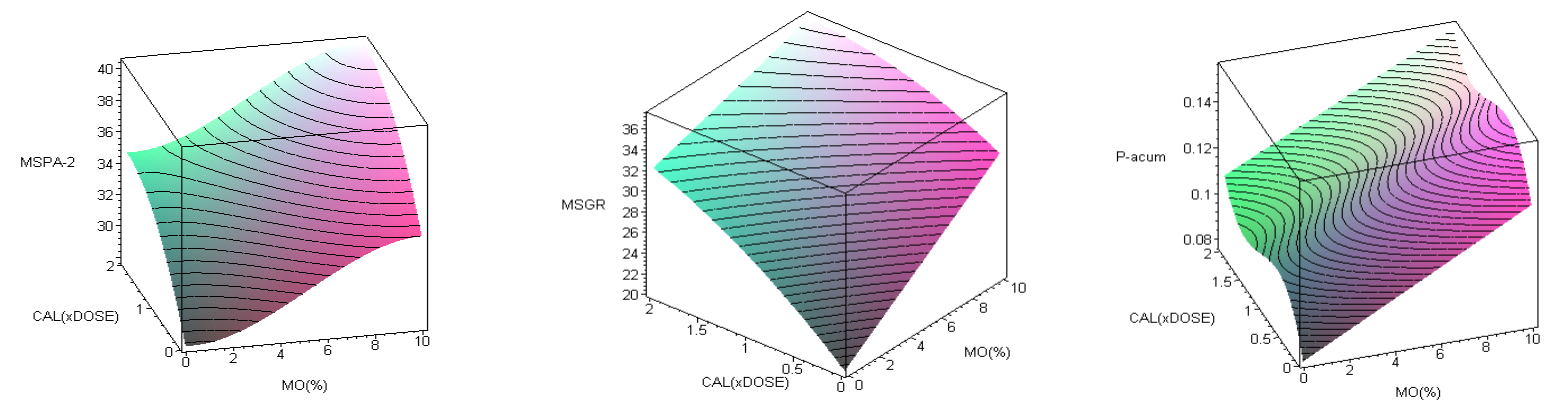

\section{LVd}
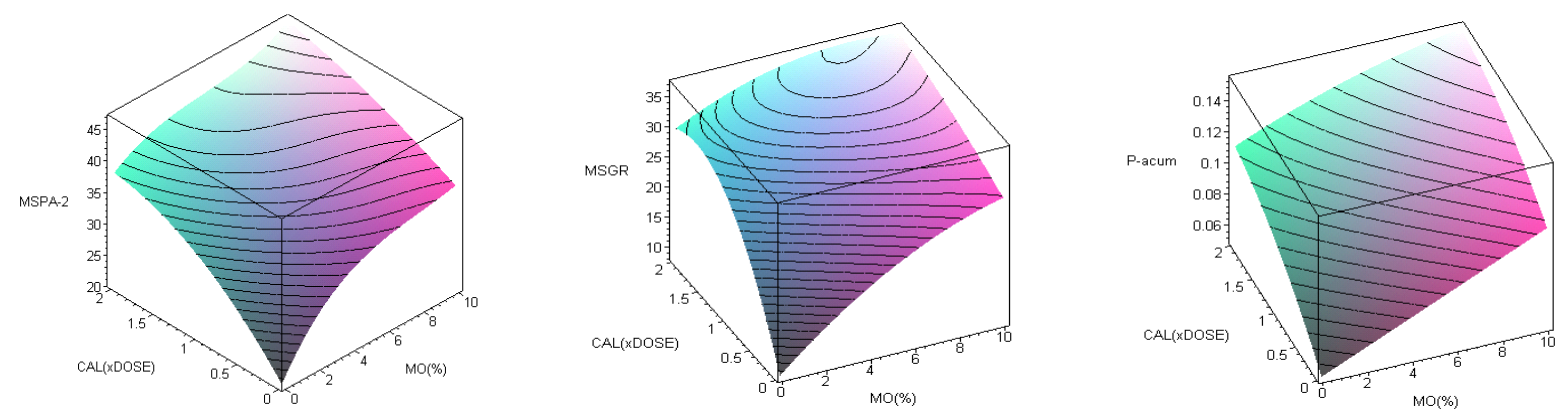

FIGURA 1 - Produção de matéria seca da parte aérea (MSPA) e de grãos (MSGR) e acúmulo total de fósforo (P-acum) na parte aérea do feijoeiro, em função da adubação orgânica e calagem (em g vaso $\left.{ }^{-1}\right)$.

Ciênc. agrotec., Lavras, v. 30, n. 4, p. 656-664, jul./ago., 2006 
TABELA 2 - Modelos ajustados para a produção de matéria seca da parte aérea (MSPA) e de grãos (MSGR) do feijoeiro e acúmulo de P na MSPA + MSGR (P-acum) em função da aplicação de esterco bovino (MO) e calcário (CAL), para os solos RQo, LVAd-1, LVAd-2, LVd.

\begin{tabular}{|c|c|c|c|}
\hline Variáveis & Solos & Modelos ajustados & $\mathbf{R}^{2}$ \\
\hline \multirow[t]{4}{*}{ MSPA } & RQo & $\begin{aligned} \mathrm{y}= & \beta_{0}+\beta_{1} \mathrm{MO}+\beta_{2} \mathrm{CAL}+\beta_{3}(\mathrm{MO})^{2}+\beta_{4}(\mathrm{CAL})^{2}+\beta_{5}(\mathrm{MO} * \mathrm{CAL}) \\
& +\beta_{6} \mathrm{MO} *(\mathrm{CAL})^{2}\end{aligned}$ & $92,11 \%$ \\
\hline & LVAd-1 & $\mathrm{y}=\beta_{0}+\beta_{1} \mathrm{MO}+\beta_{2} \mathrm{CAL}+\beta_{3}(\mathrm{CAL})^{2}+\beta_{4}(\mathrm{MO} * \mathrm{CAL})$ & $88,16 \%$ \\
\hline & LVAd-2 & $y=\beta_{0}+\beta_{1} M O+\beta_{2} C A L+\beta_{3} M O^{2}+\beta_{4} C A L^{2}+\beta_{5} M O^{3}$ & $92,71 \%$ \\
\hline & $\mathrm{LVd}$ & $\begin{aligned} \mathrm{y}= & \beta_{0}+\beta_{1} \mathrm{MO}+\beta_{2} \mathrm{CAL}+\beta_{3}(\mathrm{MO})^{2}+\beta_{4}(\mathrm{CAL})^{2}+\beta_{5}(\mathrm{MO})^{3} \\
& +\beta_{6}(\mathrm{MO} * \mathrm{CAL})+\beta_{7} \mathrm{CAL} *(\mathrm{MO})^{2}+\beta_{7}(\mathrm{CAL})^{2} * \mathrm{MO}\end{aligned}$ & $96,41 \%$ \\
\hline \multirow[t]{4}{*}{ MSGR } & RQo & $\begin{aligned} \mathrm{y}= & \beta_{0}+\beta_{1} \mathrm{MO}+\beta_{2} \mathrm{CAL}+\beta_{3}(\mathrm{MO})^{2}+\beta_{4}(\mathrm{CAL})^{2}+\beta_{5}(\mathrm{MO} * \mathrm{CAL}) \\
& +\beta_{6}(\mathrm{MO})^{2} *(\mathrm{CAL})+\beta_{7} \mathrm{MO} *(\mathrm{CAL})^{2}+\beta_{8}(\mathrm{MO})^{2} *(\mathrm{CAL})^{2}\end{aligned}$ & $71,57 \%$ \\
\hline & LVAd-1 & $\begin{aligned} \mathrm{y}= & \beta_{0}+\beta_{1} \mathrm{CAL}+\beta_{2}(\mathrm{MO})^{2}+\beta_{3}(\mathrm{CAL})^{2}+\beta_{4}(\mathrm{MO})^{3}+\beta_{5}(\mathrm{CAL})^{3} \\
& +\beta_{6}(\mathrm{CAL}) *(\mathrm{MO})^{2}+\beta_{7}(\mathrm{CAL})^{2} *(\mathrm{MO})^{2}\end{aligned}$ & $71,39 \%$ \\
\hline & LVAd-2 & $\mathrm{y}=\beta_{0}+\beta_{1} M O+\beta_{2} C A L+\beta_{3} C A L^{2}+\beta_{4} M O * C A L$ & $84,14 \%$ \\
\hline & $\mathrm{LVd}$ & $\begin{aligned} \mathrm{y}= & \beta_{0}+\beta_{1} \mathrm{MO}+\beta_{2} \mathrm{CAL}+\beta_{3}(\mathrm{MO})^{2}+\beta_{4}(\mathrm{CAL})^{2}+\beta_{5}(\mathrm{MO} * \mathrm{CAL}) \\
& +\beta_{6}(\mathrm{CAL})^{2} * \mathrm{MO}\end{aligned}$ & $90,34 \%$ \\
\hline \multirow[t]{4}{*}{ P-acum } & RQo & $\mathrm{y}=\beta_{0}+\beta_{1} \mathrm{MO}+\beta_{2} \mathrm{CAL}+\beta_{3}(\mathrm{MO})^{2}+\beta_{4}(\mathrm{CAL})^{2}+\beta_{5}(\mathrm{MO} * \mathrm{CAL})$ & $70,91 \%$ \\
\hline & LVAd-1 & $\begin{aligned} \mathrm{y}= & \beta_{0}+\beta_{1} \mathrm{MO}+\beta_{2} \mathrm{CAL}+\beta_{3}(\mathrm{MO})^{2}+\beta_{4}(\mathrm{CAL})^{2}+\beta_{5} \mathrm{MO} * \mathrm{CAL} \\
& +\beta_{6} \mathrm{CAL} *(\mathrm{MO})^{2}+\beta_{7} \mathrm{MO} *(\mathrm{CAL})^{2}+\beta_{8}(\mathrm{CAL})^{2} *(\mathrm{MO})^{2}\end{aligned}$ & $88,43 \%$ \\
\hline & LVAd-2 & $y=\beta_{0}+\beta_{1} M O+\beta_{2} C A L+\beta_{3} C A L^{2}+\beta_{4} C A L^{3}+\beta_{5} C A L^{*}(M O)^{2}$ & $69,24 \%$ \\
\hline & LVd & $\mathrm{y}=\beta_{0}+\beta_{1} \mathrm{MO}+\beta_{2} \mathrm{CAL}+\beta_{3}(\mathrm{CAL})^{2}+\beta_{4} \mathrm{CAL}^{*}(\mathrm{MO})^{2}$ & $82,33 \%$ \\
\hline
\end{tabular}

A capacidade máxima de adsorção e o P-rem são atributos que refletem o poder tampão ou fator capacidade de P no solo (MUNIZ et al., 1985). A variação do fator capacidade entre solos normalmente está associada à adsorção de $\mathrm{P}$, à mineralogia, textura e matéria orgânica do solo (BAHIA FILHO et al., 1983; MUNIZ et al., 1985). Vários autores observaram relação inversa entre o $\mathrm{P}$ absorvido pela planta e o fator capacidade do solo (BAHIA FILHO et al., 1983; FABRES et al., 1987; FERNANDES et al., 1998; HOLFORD \& MATTINGLY, 1976; MUNIZ et al., 1983). 
TABELA 3 - Estimativa dos parâmetros, significância dos modelos ajustados apresentados na Tabela 2, valores máximos para a produção de MSPA, MSGR, P-acum. e quantidades ótimas de esterco bovino (MO) e calcário (CAL), em cada solo.

\begin{tabular}{|c|c|c|c|c|}
\hline \multirow{3}{*}{ Parâmetros } & \multicolumn{4}{|c|}{ SOLOS } \\
\hline & RQo & LVAd-1 & LVAd-2 & LVd \\
\hline & \multicolumn{4}{|c|}{ MSPA } \\
\hline$\beta_{0}$ & $22,25(0,48)^{* * *}$ & $27,57(0,38)^{* * *}$ & $27,86(0,30)^{* * *}$ & $20,37(0,54)^{* * *}$ \\
\hline$\beta_{1}$ & $2,58(0,15) * * *$ & $0,76(0,05)^{* * *}$ & $-0,11(0,25) \mathrm{ns}$ & $4,81(0,36) * * *$ \\
\hline$\beta_{2}$ & $13,23(1,15) * * *$ & $7,29(0,68)^{* * *}$ & $6,89(0,53)^{* * *}$ & $16,11(1,19) * * *$ \\
\hline$\beta_{3}$ & $-0,15(0,01) * * *$ & $-1,54(0,29)^{* * *}$ & $0,19(0,06)^{* *}$ & $-0,55(0,08) * * *$ \\
\hline$\beta_{4}$ & $-4,55(0,53) * * *$ & $-0,23(0,05)^{* * *}$ & $-1,72(0,25) * * *$ & $-3,62(0,54) * * *$ \\
\hline$\beta_{5}$ & $-1,07(0,19) * * *$ & - & $-0,01(0,01)^{* *}$ & $0,02(0,00) * * *$ \\
\hline$\beta_{6}$ & $0,45(0,09) * * *$ & - & - & $-1,86(0,26) * * *$ \\
\hline$\beta_{7}$ & - & - & - & $0,09(0,02) * * *$ \\
\hline$\beta_{8}$ & - & - & - & $0,26(0,09) * * *$ \\
\hline $\begin{array}{l}\text { Pt. Max. } \\
\text { (MO,CAL) }\end{array}$ & $\begin{array}{c}38,87 \text { g.vaso } \\
(7,39 ; 2,0) \\
\end{array}$ & $\begin{array}{c}\text { 39,21g.vaso-1 } \\
(10,0 ; 1,61)\end{array}$ & $\begin{array}{c}42,66 \mathrm{~g} \cdot \text { vaso }^{-1} \\
(10,0 ; 2,00)\end{array}$ & $\begin{array}{c}\text { 42,41g.vaso }{ }^{-1} \\
(10,0 ; 2,0)\end{array}$ \\
\hline \multicolumn{5}{|c|}{ MSGR } \\
\hline$\beta_{0}$ & $14,92(1,33)^{* * *}$ & $19,73(1,08) * * *$ & $20,14(0,67)^{* * *}$ & $8,38(0,98)^{* * *}$ \\
\hline$\beta_{1}$ & $5,21(0,63) * * *$ & $27,72(5,15) * * *$ & $1,17(0,10)^{* * *}$ & $3,25(0,30) * * *$ \\
\hline$\beta_{2}$ & $19,02(3,37) * * *$ & $0,39(0,06) * * *$ & $8,47(1,19)^{* * *}$ & $28,47(2,33) * * *$ \\
\hline$\beta_{3}$ & $-0,39(0,06) * * *$ & $-20,71(7,33) * * *$ & $-1,22(0,52)^{*}$ & $-0,12(0,03) * * *$ \\
\hline$\beta_{4}$ & $-6,50(1,56) * * *$ & $-0,02(0,01) * * *$ & $-0,33(0,09)^{* * *}$ & $-8,85(1,08) * * *$ \\
\hline$\beta_{5}$ & $-6,69(1,59) * * *$ & $4,83(2,53) * * *$ & - & $-2,33(0,38) * * *$ \\
\hline$\beta_{6}$ & $0,58(0,15) * * *$ & $-0,22(0,05) * * *$ & - & $0,82(0,18) * * *$ \\
\hline$\beta_{7}$ & $2,65(0,74) * * *$ & $0,07(0,02) * * *$ & - & - \\
\hline$\beta_{8}$ & $-0,25(0,07) * * *$ & - & - & - \\
\hline $\begin{array}{c}\text { Pt. Max. } \\
\text { (MO,CAL) }\end{array}$ & $\begin{array}{c}33,38 \mathrm{~g} \text { vaso }^{-1} \\
(5,28 ; 2,0) \\
\end{array}$ & $\begin{array}{c}39,38 \text { g vaso }^{-1} \\
(10,0 ; 0,24)\end{array}$ & $\begin{array}{c}37,30 \mathrm{~g}_{\text {vaso }}{ }^{-1} \\
(10,0 ; 2,0)\end{array}$ & $\begin{array}{c}37,21 \mathrm{~g} \text { vaso }^{-1} \\
(7,79 ; 2,0)\end{array}$ \\
\hline \multicolumn{5}{|c|}{ P-acum. } \\
\hline$\beta_{1}$ & $0,0073(0,001)^{* * *}$ & $0,0120(0,0014) * * *$ & $0,0051(0,0005) * * *$ & $0,0062(0,0006)^{* * *}$ \\
\hline$\beta_{2}$ & $0,0306(0,005)^{* * *}$ & $0,0780(0,0065) * * *$ & $0,0826(0,0172) * * *$ & $0,0469(0,0072)^{* * *}$ \\
\hline$\beta_{3}$ & $-0,0003(0,001)^{* *}$ & $-0,0006(0,0001) * * *$ & $-0,0897(0,026) * * *$ & $-0,0082(0,0032)^{*}$ \\
\hline$\beta_{4}$ & $-0,0071(0,002)^{* *}$ & $-0,0287(0,0035) * * *$ & $0,0281(0,0089) * *$ & $-0,0001(0,0001)^{*}$ \\
\hline$\beta_{5}$ & $-0,0023(0,001)^{* * *}$ & $-0,0303(0,0037) * * *$ & $-0,0001(0,00004) * *$ & - \\
\hline$\beta_{6}$ & - & $0,0027(0,0004) * * *$ & - & - \\
\hline$\beta_{7}$ & - & $0,0126(0,0018) * * *$ & - & - \\
\hline$\beta_{8}$ & - & $-0,0012(0,0002) * * *$ & - & - \\
\hline $\begin{array}{c}\text { Pt Max. } \\
\text { (MO,CAL) }\end{array}$ & $\begin{array}{c}0,108 \text { g vaso }^{-1} \\
(10,0 ; 0,48)\end{array}$ & $\begin{array}{c}0,139 \text { g vaso }^{-1} \\
(10,0 ; 0,99)\end{array}$ & $\begin{array}{c}0,146 \text { g vaso }^{-1} \\
(10,0 ; 0,50)\end{array}$ & $\begin{array}{c}0,154 \mathrm{~g} \mathrm{vaso}^{-1} \\
(10,0 ; 2,0)\end{array}$ \\
\hline
\end{tabular}

***, **, *, ns: significativo a $0,1,1$ e $5 \%$ e não-significativo, respectivamente.

Ciênc. agrotec., Lavras, v. 30, n. 4, p. 656-664, jul./ago., 2006 
TABELA 4 - Coeficientes de correlação linear entre produção de matéria seca de grãos (MSGR), acúmulo de P na matéria seca da parte aérea total do feijoeiro (MSPA + MSGR) e os atributos de solo: P-rem, CMAP e P-Mehlich1.

\begin{tabular}{lcccccccc}
\hline \multirow{2}{*}{ Solos } & \multicolumn{4}{c}{ MSGR } & \multicolumn{4}{c}{ P-acumulado na MSPA + MSGR } \\
\cline { 2 - 9 } & RQo & LVAd-1 & LVAd-2 & LVd & RQo & LVAd-1 & LVAd-2 & LVd \\
\hline P-rem & $0,86^{* *}$ & $0,71^{*}$ & $0,65^{*}$ & $0,78^{*}$ & $0,91^{* *}$ & $0,69^{*}$ & $0,87^{* *}$ & $0,89^{* *}$ \\
CMAP & $-0,85^{* *}$ & $\mathrm{~ns}$ & $\mathrm{~ns}$ & $-0,64^{*}$ & $-0,86^{* *}$ & $\mathrm{~ns}$ & $-0,84^{* *}$ & $-0,76^{*}$ \\
P-Mehlich1. & $0,78^{*}$ & $0,70^{*}$ & $0,74^{*}$ & $0,72^{*}$ & $0,72^{*}$ & $0,73^{*}$ & $0,70^{*}$ & $\mathrm{~ns}$ \\
\hline
\end{tabular}

**, *e ns: significativo a 1 e $5 \%$ e não-significativo, respectivamente.

\section{CONCLUSÕES}

A adição de esterco bovino e calcário, alterando os atributos ligados ao fator capacidade de $\mathrm{P}$ no solo e sua maior disponibilidade, afetou positivamente a absorção de fósforo pelo feijoeiro e, conseqüentemente, promoveu um maior acúmulo do nutriente na parte aérea total e incremento na produção de grãos.

\section{REFERÊNCIAS BIBLIOGRÁFICAS}

ALVAREZ, V. H. Avaliação da fertilidade do solo: superfícies de resposta: modelos aproximativos para expressar a relação fator-resposta. Viçosa: UFV, 1994. 75 p.

ALVAREZ, V. H.; FONSECA, D. M. Definição de doses de fósforo para determinação da capacidade máxima de adsorção de fosfatos e para ensaios de casa de vegetação. Revista Brasileira de Ciência do Solo, Campinas v. 14, n. 1, p. 48-55, jan./abr. 1990.

ALVAREZ, V. H.; NOVAIS, R. F.; DIAS, L. E.; OLIVEIRA, J. A. Determinação e uso do fósforo remanescente. Boletim Informativo da Sociedade Brasileira de Ciência do Solo, Viçosa, v. 25, n. 1, p. 27-32, jan./mar. 2000.

ANDRADE, A. T.; FERNANDES, L. A.; FAQUIN, V. Organic residue, limestone gypsum and phosphorus adsorption by lowland soils. Scientia Agrícola, Piracicaba, v. 59, n. 2, p. 349-355, abr./jun. 2002.

BAHIA FILHO, A. F. C.; BRAGA, J. M.; RESENDE, M.; RIBEIRO, A. C. Relação entre adsorção de fósforo e componentes mineralógicos da fração argila de latossolos do Planalto Central. Revista Brasileira de Ciência do Solo, Campinas, v. 7, n. 3, p. 221-226, set./dez. 1983.
BRAGA, J. M.; DEFELIPO, B. V. Determinação espectrofotométrica de fósforo em extratos de solo e material vegetal. Revista Ceres, Viçosa, v. 21, n. 113, p. 7385, jan./fev. 1974.

COMISSÃO DE FERTILIDADE DO SOLO DO ESTADO DE MINAS GERAIS. Recomendação para o uso de corretivos e fertilizantes em Minas Gerais: $5^{\text {a }}$ aproximação. Viçosa, 1999.359 p.

DAY, P. R. Particle fractionation and particle-size analysis. In: BLACK, C. A. (Ed.). Methods of soil analysis. Madison: ASA, 1965. v. 1, p. 545-566.

EMPRESA BRASILEIRA DE PESQUISA AGROPECUÁRIA. Centro Nacional de Pesquisa de Solos. Sistema brasileiro de classificação de solos. Brasília, DF: Embrapa Produção de informação; Rio de Janeiro: Embrapa Solos, 1999. $412 \mathrm{p}$.

EMPRESA BRASILEIRA DE PESQUISA AGROPECUÁRIA. Serviço Nacional de Levantamento e Conservação de Solo. Manual de métodos de análise de solo. Rio de Janeiro: Ministério da Agricultura, 1997. 212 p.

FABRES, A. S.; NOVAIS, R. F.; NEVES, J. C. L.; BARROS, N. F.; CORDEIRO, A. T. Níveis críticos de diferentes frações de fósforo em plantas de alface cultivado em diferentes solos. Revista Brasileira de Ciência do Solo, Campinas, v. 11, n. 1, p. 51-57, jan./abr. 1987.

FAGERIA, N. K.; SANTOS, A. B. Adubação fosfatada para o feijoeiro em solo de várzea. Revista Brasileira de Engenharia Agrícola e Ambiental, Campina Grande, v. 2, n. 2, p. 124-127, maio/ago. 1998. 
FAQUIN, V.; ANDRADE, C. A. B.; FURTINE NETO, A. E.; ANDRADE, A. T.; CURI, N. Resposta do feijoeiro à aplicação de calcário em solos de várzea do sul de Minas Gerais. Revista Brasileira de Ciência do Solo, Viçosa, v. 22, n. 4, p. 651-660, out./dez. 1998.

FERNANDES, L. A.; FAQUIN, V.; FURTINI, A. E.; CURI, N. Formas de fósforo em solos de várzea e biodisponibilidade para o feijoeiro. Pesquisa Agropecuária Brasileira, Brasília, v. 37, n. 3, p. 373-383, mar. 2002.

FERNANDES, L. A.; FURTINE NETO, A. E.; CURI, N.; GUEDES, G. A. A.; LIMA, J. M. Fósforo e atividade da fosfatase ácida em plantas de feijoeiro. Pesquisa Agropecuária Brasileira, Brasília, v. 35, n. 5, p. 769-778, maio 1998.

FREIRE, J. C.; RIBEIRO, M. A. V.; BAHIA, V. G.; LOPES, A. S.; AQUINO, L. H. Resposta do milho cultivado em casa de vegetação a níveis de água em solos da região de Lavras (MG). Revista Brasileira de Ciência do Solo, Campinas, v. 4, n. 1, p. 5-8, jan./abr. 1980.

HOLFORD, I. C. R.; MATTINGLY, G. E. G. Phosphate adsorption and availability plant of phosphate. Plant and Soil, Dordrecht, v. 44, p. 377-389, 1976.

KHURI, A. I.; CORNELL, J. A. Response surfaces designs and analyses. 2. ed. New York: [s.n.], 1996. 510 p.

KLUG, H. P.; ALEXANDER, L. E. X-ray diffraction procedures for polycrystalline and amorphous materials. New York: J. Wiley, 1974. 716 p.

MALAVOLTA, E.; VITTI, G. C.; OLIVEIRA, S. A. de. Avaliação do estado nutricional das plantas: princípios e aplicações. Piracicaba: POTAFOS, 1997. 210 p.

MARSCHNER, H. Mineral nutrition of higher plants. 2. ed. London: Academic, 1995. 889 p.

MEHRA, O. P.; JACKSON, N. L. Iron oxide removal from soils and clays by a dithionite-citrate system buffered with sodium bicarbonate. Clays and Clays Minerals, Lawrence, v. 3, p. 317-327, 1960.
MEURER, E. J.; RHENHEIMER, D.; BISSANI, C. A. Fenômeno de sorção em solos. In: MEURER, J. E. (Ed.). Fundamentos de química do solo. 2. ed. Porto Alegre: Gênesis, 2004. p. 131-179.

MUNIZ, A. S.; NOVAIS, R. F.; BARROS, N. F.; NEVES, J. C. L. Nível crítico de fósforo na parte aérea da soja como variável do fator capacidade de fósforo. Revista Brasileira de Ciência do Solo, Campinas, v. 9, n. 3, p. 237-244, set./dez. 1985.

NOVAIS, R. F.; SMYTH, T. J. Fósforo em solo e planta em condições tropicais. Viçosa: UFV/DPS, 1999. 399 p.

OLIVEIRA, I. P.; THUNG, M. D. T.; KLUTHCOUSKI, J.; AIDAR, H.; CARVALHO, J. R. P. Avaliação de cultivares de feijão quanto à eficiência no uso de fósforo. Pesquisa Agropecuária Brasileira, Brasília, v. 22, n. 1, p. 39-45, jan. 1987.

PARRA, M. S.; MIRANDA, G. M. Uso de fertilizantes na cultura do feijoeiro. In: INSTITUTO AGRONÔMICO DO PARANA. Uso de fertilizantes na agricultura. Londrina: IAPAR, 1980. p. 55-60. (IAPAR, Circular, 16).

RAIJ, B. van; QUAGGIO, J. A.; CANTARELLA, H.; FERREIRA, M. E.; LOPES, A. S.; BATAGLIA, O. C. Análise química do solo para fins de fertilidade. Campinas: Fundação Cargil, 1987. 170 p.

R DEVELOPMENT CORE TEAM. R language and environment for statistical computing. Vienna, 2004. Disponível em: $\langle$ http://Www.R-project.org $\rangle$. Acesso em: 25 dez. 2004.

SCHWERTMANN, U. Differenzierung der eisenoxide des bondes durch extraktion mit ammonium-oxalat-losung. Zeitschrift fuer Pflanzernahrung, Berlin, v. 105, n. 4, p. 194-202, 1964.

SOUZA, R. F. de. Dinâmica de fósforo em solos sob influência da calagem e adubação orgânica, cultivados com feijoeiro. 2005. 141 p. Tese (Doutorado em Solos e Nutrição de Plantas) - Universidade Federal de Lavras, Lavras, 2005.

TAIZ, L.; ZEIGER, E. Fisiologia vegetal. 3. ed. Porto Alegre: Artmed, 2004. 719 p. 